\title{
Minimizing the Number of One-Paths in BDDs by an Evolutionary Algorithm
}

\author{
Mario Hilgemeier Nicole Drechsler Rolf Drechsler \\ Institute of Computer Science \\ University of Bremen \\ 28359 Bremen, Germany \\ $\{m h, n d, r d\} @$ informatik.uni-bremen.de
}

\begin{abstract}
Ordered binary decision diagrams (BDDs) are used in VLSI CAD, especially for the canonical representation of Boolean functions. In the last decade, the method of choice for optimizing this data structure was minimizing the number of nodes in the associated graph. However, recent works have shown that the number of paths is also important.

In this work, minimizing the number of one-paths of BDDs is accomplished by an evolutionary algorithm (EA) acting on the permutation of variables. The optimal operator weights for the EA were determined by a parameter study. Experimental results demonstrate the efficiency of our approach.
\end{abstract}

\section{Introduction}

Ordered binary decision diagrams (BDDs) are known to be canonical representations of Boolean functions by a directed graph $[3,4]$. One-paths are those routes through the BDD that start at a root and terminate in a logical 1.

In most cases, the minimal number of nodes is the optimization objective. However, one-paths are important for certain applications in the field of VLSI CAD. These applications include:

- Formal verification using SAT-solving: The number of steps necessary to solve a SAT problem is directly related to the number of paths in the corresponding BDD [14].

- Logic synthesis: The number of paths influences the minimization process $[12,17]$. A minimized disjoint-sum-of-product representation can directly be extracted from the BDD [9] and leads to smaller circuits.

Currently applied typical heuristic methods for minimizing the number of one-paths like modified sifting (MS) [8] run very fast on certain Boolean functions. But MS can be surpassed by evolutionary algorithms if one is willing to invest more computing time.

Evolutionary algorithms (EAs) excel in problem areas where no straightforward solution method with low computational complexity is known. EAs use a population of solutions that can evolve by producing new offspring using mutation and genetic recombination. The best individuals of that population survive and can have new offspring. Thus a convergence towards good solutions is attained. For example, EAs were successful in VLSICAD [5] and embedded system design [6].

The one-path minimization problem in a BDD is a computationally hard problem that can be attacked by an EA.
That EA searches for an optimal permutation of the variable order in the BDD. This work shows that an EA can substantially reduce the number of one-paths in BDDs.

The paper is structured as follows: Section 2 explains BDDs and the MS algorithm. The EA is described in detail in Section 3. In Section 4, three promising operator combinations of a standard EA are selected. The most successful of these three is then compared to the results of MS. Section 5 concludes the paper and summarizes the results.

\section{Preliminaries}

This section introduces some basics concerning BDDs, onepaths, and reviews a state-of-the-art heuristic to minimize the number of one-paths that will be used later for comparison with the EA.

\subsection{Binary Decision Diagrams}

BDDs [3] are directed acyclic graphs where a Shannon decomposition is carried out in each node. Each node has a Boolean variable attached to it from the corresponding logical expression that describes the Boolean function $f: B^{n} \rightarrow B^{m}$. Depending on the value of the variable, the path is chosen; in Figures 1 and 2, a dashed line represents a logical 0 value and a logical 1 is shown as a straight line. In these examples, $n=5$ and $m=1$. Note that there are $m$ root nodes. In the case of $m>1$, one speaks of a shared BDD because $f=\left(f_{1}, \ldots, f_{m}\right)$ is composed of $m$ functions $f_{i}: B^{n} \rightarrow B, \quad 1<i \leq m$.

If a BDD contains no isomorphic sub-graphs and no vertices with both outgoing edges pointing to the same node, that BDD is called reduced. Following an ordered BDD down from its root node, each Boolean variable value is met at most once and in the same order along each path.

Typically, a variable is ascribed to more than one node. In this paper, we restrict "BDD" to mean reduced ordered binary decision diagrams. It follows that the nodes belonging to the same variable can be arranged in horizontal levels.

The BDDs terminate either in logical 0 or 1 . One-paths are those routes through the BDD that start at the root and terminate in the node representing logical 1.

The number of nodes necessary to represent the BDD for the Boolean function is dependent on the variable (i.e. level) order. The following example will show the effects of variable ordering on the number of nodes of a BDD and especially the number of one-paths. The number of one-paths is denoted by $P_{1}$, the number of zero-paths by $P_{0}$.

Example 1. Consider the BDDs in Figures 1 and 2 [7]. Both BDDs represent the same Boolean function 


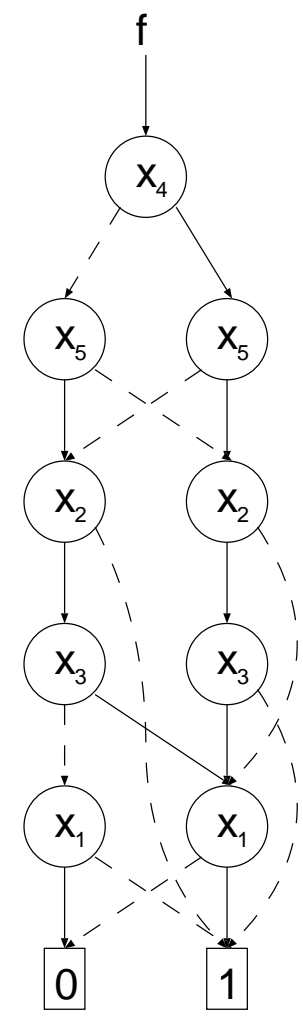

Figure 1: BDD of $f$ with minimized nodes

$f\left(x_{1}, x_{2}, x_{3}, x_{4}, x_{5}\right)$ which is defined by

$f=x_{1} \overline{\left(x_{4} \oplus x_{5}\right)}+\overline{x_{2}}\left(x_{4} \oplus x_{5}\right)+x_{1} x_{3}+\overline{x_{1}} x_{2} \overline{x_{3}}$.

Figure 1 shows 11 nodes. This BDD corresponds to the variable order $\left(x_{4}, x_{5}, x_{2}, x_{3}, x_{1}\right)$; it has $P_{0}=8$ and $P_{1}=12$. The logically equivalent BDD in Figure 2 has the variable order $\left(x_{2}, x_{3}, x_{4}, x_{5}, x_{1}\right)$. It has more nodes (12), but only 9 one-paths and 5 zero-paths.

In the above example it has been demonstrated that the number of one-paths does not have to be minimal when the number of nodes is minimized and vice versa.

\subsection{Modified Sifting}

Sifting [15] is a well-known algorithm for minimizing the number of nodes in a BDD. It works the following way: while the other variables remain in their positions, a single variable is shifted to all possible positions. Then the best of these positions - namely the one with the minimal number of nodes - is accepted for that variable. This is done once for all variables.

Modified sifting (MS) [8] works like the sifting algorithm for minimizing the number of nodes, but in this case the acceptance criterion is that $P_{1}$ has to be minimal.

\section{Evolutionary Algorithm}

In this section, the employed standard EA with sexual reproduction, recombination and mutation and its associated operators is introduced. This standard EA is only modified with respect to the probabilities of these operators.

The search for the minimal number of one-paths for the BDD representations of a given Boolean function is analo-

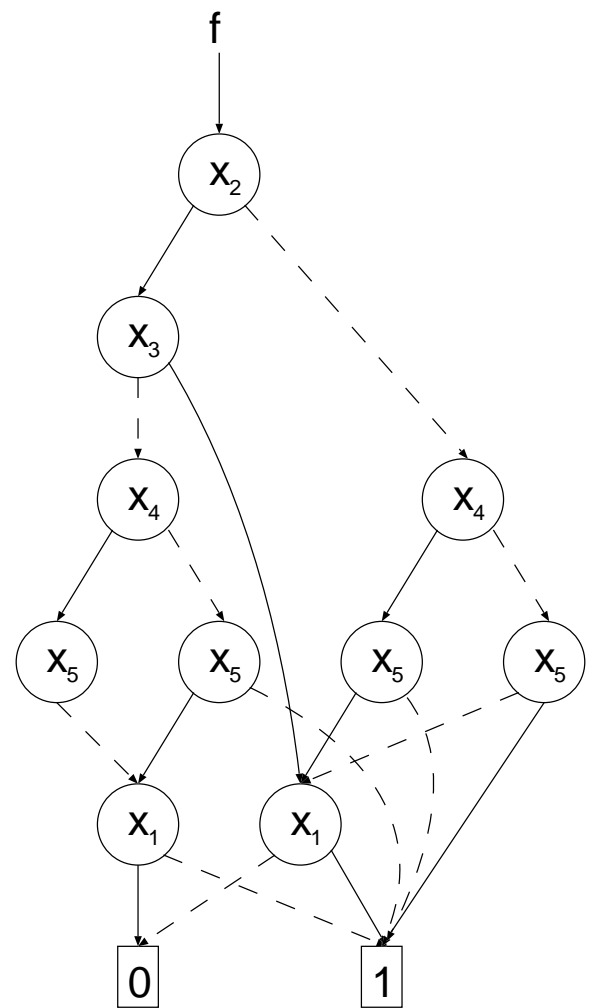

Figure 2: BDD of $f$ with minimized paths

gous to the search for a permutation of the node indices.

We will first give details of coding, fitness evaluation, and operators. Then the evolutionary algorithm is sketched.

\subsection{Coding and Fitness Evaluation}

An individual of the population is described by a permutation of the variable indices of the BDD. For each individual, this permutation is stored in an index list. This index list constitutes the chromosome of the individual.

During the minimum search for a single Boolean function, $P_{1}$ is the fitness function for each individual.

\subsection{Operators}

We now briefly describe the operators that will be used by the EA.

The reproduction includes crossover operators that are specially designed for recombination of permutations. Onepoint crossover does not work for permutations because this leads to duplicates and omissions (except in the case where both permutations contain the same set of variable indices on corresponding sides of the cut). Therefore crossover operators employ "repair mechanisms" that make sure that the permutation remains valid or use algorithms that always produce valid permutations. Typically, these operators produce two children from two parents.

After reproduction, a single mutation operator might be applied according to the given probabilities. A mutation operator acts on a single child. That is, the two children from a reproduction may be mutated by different operators.

In other words, a new child might have been subject to one crossover, to one mutation, or both. 


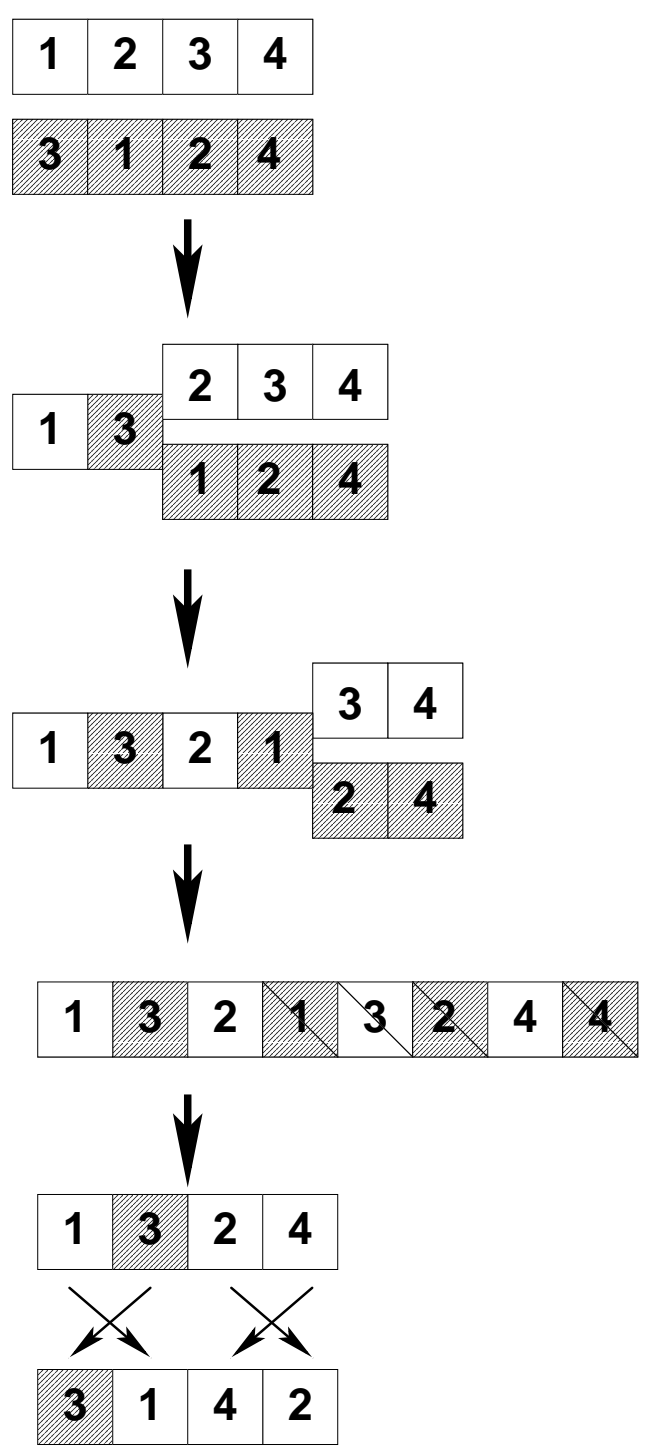

Figure 3: Example for the MERGE operator

Reproduction and mutation are invoked according to the given probabilities. Reproduction operators used are PMX, CX, MERGE, INV and REP. Mutation operators include MUTP1, MUTP2, and MUTPD. These operators will be described now.

- PMX (partially matched crossover) [13] respects the absolute position of each variable index. PMX sets two "crossover points" in the permutation between which the index order remains the same as in the parent. For the other positions in the permutation, a substitution mechanism guarantees that each variable index appears exactly once in each permutation.

- CX (cycle crossover) [11] makes use of cycles in the permutation to avoid double or missing variable indices after the crossover.

- MERGE produces the first child in the following way. Alternating between the parents, MERGE takes one variable index from each parent (in the order they appear in the parents) until the permutation length is reached. After doing this, MERGE checks from left to right if an index has been used already. If this is the case, that index number is removed.

The second child is produced by exchanging the indices of even and odd positions in the child permutation (see Example 2 below).

- The inversion operator INV inverts the order of the index list for a randomly chosen part of the chromosome. It produces one child from each parent.

- The reproduction operator REP reads the index list forward. This means that no genetic change is introduced by this operator. It only copies individuals. It produces two children that are identical to their parents.

- The mutation operator MUTP1 selects two random positions in the list, and exchanges their contents.

- MUTP2 is MUTP1 applied twice to the same individual.

- MUTPD exchanges randomly chosen adjacent positions in the permutation.

All operators produce only valid offspring. As an example for the MERGE operator, consider Figure 3.

Example 2. Let parent 1 be the permutation $(1,2,3,4)$ and parent 2 be the permutation $(3,1,2,4)$ (see Figure 3). Now imagine these as the two parts of an open zipper. When the zipper is closed, the two halves merge, yielding $(1,3,2,1,3,2,4,4)$.

Working from left to right, the redundant variable indices are cancelled (boxes diagonally striked out in Figure 3). So the first child becomes $(1,3,2,4)$.

Now the second child is generated by exchanging even and odd positions (crossed arrows in Figure 3). Numbers 1 and 3 as well as 2 and 4 are exchanged, yielding $(3,1,4,2)$.

\subsection{Algorithm}

The flow of the EA is sketched in Figure 4. At the beginning, individuals are initialized with random permutations of the variable indices. The population size is three times the number of inputs of the Boolean function being minimized, but not greater than 120. Each individual is assigned

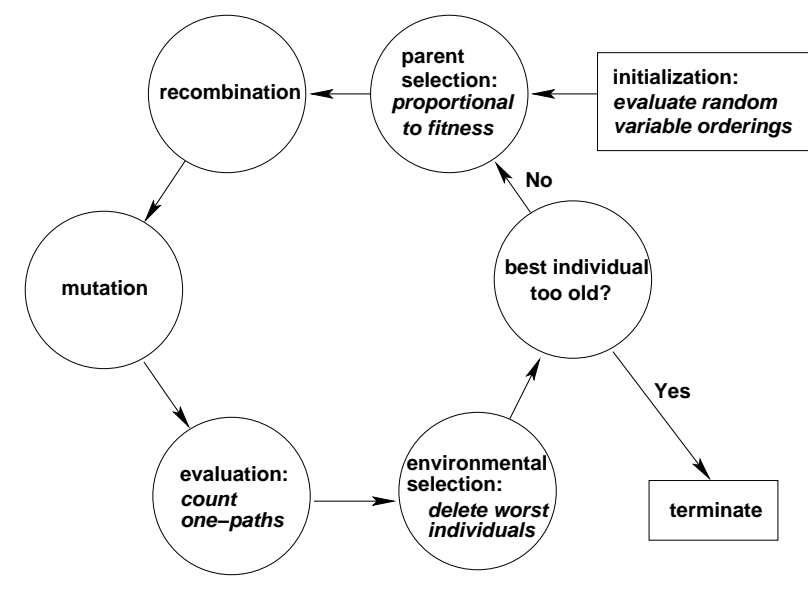

Figure 4: Sketch of the EA 
Table 1: Operator weightings with their EA results

\begin{tabular}{|c|c|c|c|c|c|c|c|c|}
\hline \multirow[b]{2}{*}{ operator } & \multicolumn{8}{|c|}{ weighting \# } \\
\hline & 1 & 2 & 3 & 4 & 5 & 6 & 7 & 8 \\
\hline PMX & 0.9510 & 0.9216 & 0.9510 & 0.9216 & 0.98 & 1.00 & 0.98 & 0.98 \\
\hline $\mathrm{CX}$ & 0.0000 & 0.0196 & 0.0000 & 0.0196 & 0.00 & 0.00 & 0.00 & 0.00 \\
\hline MERGE & 0.0000 & 0.0196 & 0.0000 & 0.0196 & 0.00 & 0.00 & 0.00 & 0.00 \\
\hline INV & 0.0245 & 0.0196 & 0.0245 & 0.0196 & 0.01 & 0.00 & 0.01 & 0.01 \\
\hline REP & 0.0245 & 0.0196 & 0.0245 & 0.0196 & 0.01 & 0.00 & 0.01 & 0.01 \\
\hline MUTP1 & 0.05 & 0.33 & 0.33 & 0.05 & 0.05 & 0.05 & 0.05 & 0.02 \\
\hline MUTP2 & 0.05 & 0.33 & 0.33 & 0.05 & 0.05 & 0.05 & 0.05 & 0.02 \\
\hline MUTPD & 0.00 & 0.33 & 0.33 & 0.00 & 0.00 & 0.00 & 0.05 & 0.00 \\
\hline \multicolumn{9}{|l|}{ result } \\
\hline$\Sigma$ nodes & 11968 & 12481 & 13861 & 14344 & 13284 & 12663 & 11985 & 15283 \\
\hline$\Sigma P_{1}$ & 29235 & 31139 & 29406 & 29258 & 29218 & 29492 & 29340 & 29785 \\
\hline$\Sigma \sec$ & 1757.95 & 1781.84 & 1748.23 & 1856.52 & 1830.61 & 1720.19 & 1720.70 & 1756.22 \\
\hline$\Sigma$ gener. & 9494 & 9810 & 9389 & 10313 & 10156 & 9266 & 9294 & 9712 \\
\hline \multirow[b]{2}{*}{ operator } & \multicolumn{8}{|c|}{ weighting \# } \\
\hline & 9 & 10 & 11 & 12 & 13 & 14 & 15 & 16 \\
\hline PMX & 0.98 & 0.98 & 0.98 & 0.98 & 0.00 & 0.00 & 0.36 & 0.00 \\
\hline $\mathrm{CX}$ & 0.00 & 0.00 & 0.00 & 0.00 & 0.98 & 0.00 & 0.32 & 0.48 \\
\hline MERGE & 0.00 & 0.00 & 0.00 & 0.00 & 0.00 & 0.98 & 0.32 & 0.48 \\
\hline INV & 0.01 & 0.01 & 0.01 & 0.01 & 0.01 & 0.01 & 0.01 & 0.01 \\
\hline REP & 0.01 & 0.01 & 0.01 & 0.01 & 0.01 & 0.01 & 0.01 & 0.01 \\
\hline MUTP1 & 0.07 & 0.10 & 0.06 & 0.08 & 0.07 & 0.07 & 0.07 & 0.07 \\
\hline MUTP2 & 0.07 & 0.10 & 0.06 & 0.08 & 0.07 & 0.07 & 0.07 & 0.07 \\
\hline MUTPD & 0.00 & 0.00 & 0.00 & 0.00 & 0.00 & 0.00 & 0.00 & 0.00 \\
\hline result & & & & & & & & \\
\hline$\Sigma$ nodes & 12972 & 13299 & 12834 & 12542 & 16561 & 12863 & 11394 & 13240 \\
\hline$\Sigma P_{1}$ & 29191 & 29392 & 29269 & 31569 & 31964 & 31981 & 31392 & 29893 \\
\hline$\Sigma \sec$ & 1706.35 & 1732.86 & 1669.44 & 1922.72 & 2041.20 & 1763.46 & 1686.11 & 1717.26 \\
\hline$\Sigma$ gener. & 9580 & 9556 & 9119 & 10105 & 10132 & 11186 & 9797 & 9550 \\
\hline
\end{tabular}

a fitness according to the number of one-paths of the corresponding BDD.

After initialization the evolutionary loop is started. Fitness-proportional selection [11], i.e. roulette wheel selection, determines which parents will produce children. In each generation, the number of children is half the population size.

Recombination and mutation operators are chosen randomly according to the specified set of operator probabilities. The fitness evaluation consists of the counting of the one-paths of the children. To keep the population constant, the worst third of this bigger population (parents + children) is discarded during the environmental selection before the next generation.

The termination condition means that the best individual has survived for a long time and no improvements were found during its lifetime.

\section{Experiments}

To tune and test the EA, a number of experiments was conducted with various benchmarks functions. These benchmark functions were chosen as a representative set of easy and hard problems. Moreover, the benchmarks are mostly not too expensive, computationally, so that the set of benchmarks can be run in reasonable time.

\subsection{Qualities}

In this section we give some arguments why we deem the chosen set of benchmark functions representative.

The sizes of the 38 benchmark functions vary over a wide range. They have between 5 and 54 inputs and between 1 and 41 outputs (for a detailed list see Table 3 ). From the MS algorithm, the values for $P_{1}$ are known to vary over more than three orders of magnitude.

\subsection{Computing Environment}

The tests for the EA as well as the reference runs for the MS algorithm were done on a Linux machine equipped with an AMD Athlon XP2200+ processor $(1.8 \mathrm{GHz}, 256 \mathrm{kB}$ cache) and 512 MB RAM.

For the BDD representation, CUDD [16] was used. The EA is based on the $\mathrm{C}++$ library for evolutionary algorithms GAME [10] (version 2.3). Test control programs were written in Python. 


\subsection{Parameter Selection}

In order to assess the usefulness of the EA operators (see Section 3.2) for our set of benchmarks, a good, balanced set of operators is sought. Such a set of operator probabilities is called a weighting. To find a good operator set, sixteen different weightings were tested, e.g. with PMX, CX, and MERGE of nearly equal probability, etc.

A simple measure of goodness for an operator weighting is the sum over all $P_{1}$ values. In this way, benchmarks with larger absolute improvements of $P_{1}$ influence the sum stronger. We considered this a desirable effect because these more complex functions are the intended application area.

The final result of this parameter exploration is shown in Table 1. The three best results for the sum of $P_{1}$ are shown in boldface. The most promising operator weighting is \#9.

This operator weighting of the EA was used for comparison with MS. Since running the EA with a specific parameter set over all 38 benchmarks needed about half an hour with the given hardware configuration, each weighting was tested only once during the selection process.

\subsection{EA Performance}

The performance of the selected EA was tested by running it 25 times, each time over all the benchmarks.

The different value spreads for nodes and $P_{1}$ are shown graphically in Figure 5. Each benchmark is represented by a dot. For the 25 runs of each benchmark with the finally selected operator weightings, median (dot) and value spread (bars) are shown. The horizontal bars show the range of the resulting node numbers of the BDD of each benchmark. The vertical bars correspond to the range of results in $P_{1}$. Note that in this plot of one-paths versus nodes both axes are logarithmically scaled.

Figure 5 shows the wide range of problem sizes and the rough correlation between the length of one-paths and the number of nodes.

The complete statistics for EA (Table 2) shows that the value range of found $P_{1}$ minima is quite small for most functions. However, the value spread becomes larger when the $P_{1}$ values increase. Examples for this are the first two (s1196, s1238) and the last three functions (cordic, misex3, $s e q$ ). Table 2 serves as a value table for Figure 5 where this effect shows: the functions with smaller $P_{1}$ rarely have visible $P_{1}$ error bars. In this logarithmic diagram only functions with large $P_{1}$ have visible error bars which means that their percentual value spread relative to the median is greater.

\subsection{Comparison to Modified Sifting}

We now compare our EA to the state-of-the-art heuristic for one-path minimization, MS. First, we will have a look at a graphic representation of the differences. After that, the details will be discussed using Table 3 .

In Figure 6, the differences between our EA and MS are shown. For each best benchmark result of these two algorithms, the difference of nodes (abscissa value) and the negative difference of $P_{1}$ (ordinate value) was computed and plotted. Note that the ordinate is logarithmically scaled (about four orders of magnitude).

Because of the logarithmic scale, functions with onepath differences of zero are not shown. It is worth men-
Table 2: Descriptive statistics for the EA

\begin{tabular}{|l||r|r|r|r|}
\hline \multicolumn{1}{|l||}{ function } & min. & mean & median & max. \\
\hline s1196 & 2508 & 2626.92 & 2673 & 2783 \\
s1238 & 2508 & 2621.40 & 2650 & 2757 \\
s1488 & 352 & 352.00 & 352 & 352 \\
s1494 & 352 & 352.12 & 352 & 353 \\
s208 & 53 & 53.04 & 53 & 54 \\
s27 & 16 & 16.00 & 16 & 16 \\
s298 & 70 & 70.00 & 70 & 70 \\
s344 & 330 & 331.80 & 330 & 348 \\
s349 & 330 & 332.16 & 330 & 351 \\
s382 & 230 & 231.32 & 230 & 239 \\
s386 & 57 & 57.56 & 57 & 64 \\
s400 & 230 & 230.64 & 230 & 236 \\
s444 & 230 & 232.32 & 231 & 239 \\
s510 & 153 & 154.88 & 154 & 163 \\
s526 & 156 & 159.88 & 159 & 172 \\
s526n & 156 & 158.60 & 158 & 168 \\
s641 & 1444 & 1498.36 & 1497 & 1580 \\
s713 & 1447 & 1497.04 & 1488 & 1596 \\
s820 & 146 & 146.04 & 146 & 147 \\
s832 & 146 & 146.12 & 146 & 149 \\
alu4 & 1372 & 1372.00 & 1372 & 1372 \\
b12 & 60 & 60.04 & 60 & 61 \\
clip & 214 & 214.00 & 214 & 214 \\
inc & 27 & 27.00 & 27 & 27 \\
majority & 5 & 5.00 & 5 & 5 \\
misex1 & 34 & 34.00 & 34 & 34 \\
misex2 & 29 & 29.56 & 30 & 30 \\
rd53 & 35 & 35.00 & 35 & 35 \\
rd73 & 147 & 147.00 & 147 & 147 \\
rd84 & 294 & 294.00 & 294 & 294 \\
sao2 & 97 & 97.12 & 97 & 98 \\
t481 & 841 & 867.88 & 841 & 1009 \\
xor5 & 16 & 16.00 & 16 & 16 \\
5xp1 & 79 & 79.00 & 79 & 79 \\
9 sym & 148 & 148.00 & 148 & 148 \\
cordic & 8332 & 11349.12 & 11044 & 13218 \\
misex3 & 1976 & 1991.28 & 1987 & 2031 \\
seq & 1744 & 1753.20 & 1752 & 1769 \\
\hline & & & & \\
S & & & & \\
\hline
\end{tabular}

tioning in this context that in no case the EA was worse than MS.

Many values cluster near a zero node difference while being better in the one-path difference. For these benchmarks, $P_{1}$ can be improved at little or no increase in node number. It is noteworthy that most of the time (i.e. all dots left of zero node difference), an improvement in $P_{1}$ goes with a decrease in node number (contrary to Example 1). For greater differences in one-paths the spread in node difference becomes larger.

For a closer inspection of benchmark results we now use Table 3. For each function, the results of the two different algorithms are shown in one row.

The upper, larger part of Table 3 contains the ISCAS89 benchmarks functions $[2,1]$ considered in [8]. Separated 


\section{medians with minimum and maximum for EA}

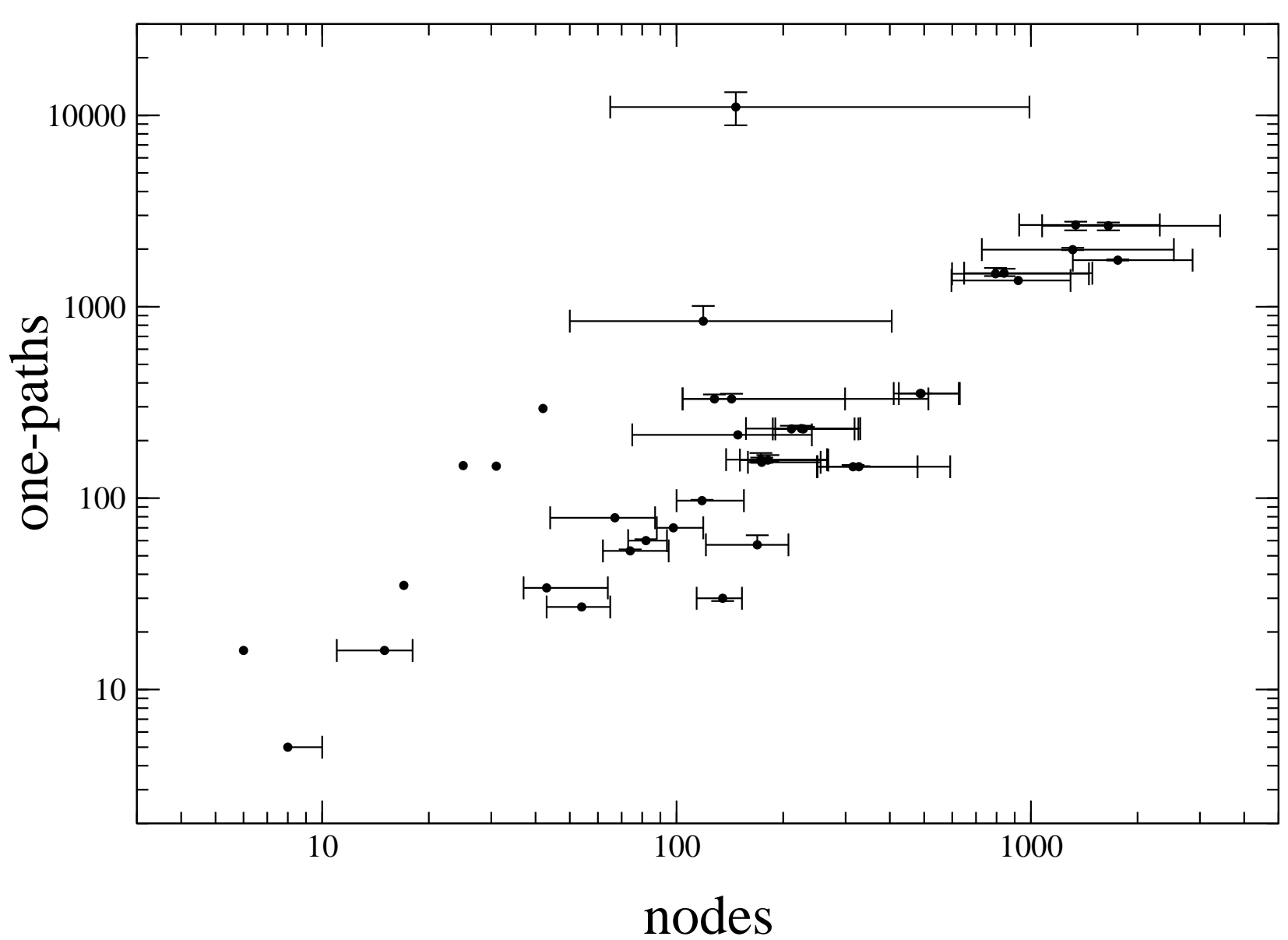

Figure 5: Qualities of benchmark set. Plot of $P_{1}$ versus nodes.

by a horizontal line and below these are functions that have been used in a recent paper [9]. The lower, smaller part of Table 3 gives three benchmarks that were deemed especially difficult. Each of the two parts of Table 3 has a statistic section at its bottom.

For comparison, the one-path minimization results from the MS algorithm [8] are shown in the three columns labeled nodes, $P_{1}$, and sec (CPU run-time in seconds).

The results of our EA shown in Table 3 are the minima of the 25 benchmark runs. The run-time given is that of one single run. If there were several runs with minimal $P_{1}$, the run with the smallest number of nodes was chosen. For 20 functions, the best EA results were better than MS in node number; in this cases, the node number was printed in boldface. It is remarkable that for these runs the number of generations is often also small: a good permutation was found early.

Normally, the EA runs much longer than MS, as was expected. But it should be mentioned that functions exist where this is not the case. The seq function is such a case which proved difficult for MS. Here the EA is superior not only in $P_{1}$ but also in run-time.

In the 38 benchmarks considered, the EA improves on the one-path minima found by MS in 25 cases (66\%). In all other cases EA was as good as MS, concerning minimal
$P_{1}$. In 17 cases (45\%) the EA additionally improved on the number of nodes in the BDD.

Improvements by the EA in comparison to MS amounted to up to $72 \%$ with a mean improvement of $8.4 \%$.

The EA is a reliable algorithm for the $P_{1}$ minimization problem. This is exemplified by the fact that the median $P_{1}$ values for the EA (see Table 2 ) are never worse than the results of MS in Table 3. This means that about every second run of this EA is better than MS for a given benchmark; one has to run only a handful of EA attempts to get an improvement over MS. And the best $P_{1}$ results of our EA are always better or equal to MS (boldface in Table 3 ).

\section{Conclusions}

In this paper, the number of one-paths of various Boolean functions was minimized by an EA. The most promising combination from a set of different operator weightings for that EA was chosen. This most successful parameter setting predominantly used partially matched crossover (PMX) as recombination operator. The EA with this parameter setting always found the $P_{1}$ minima of the MS method, most of the time improving on these. 
EA minus MS

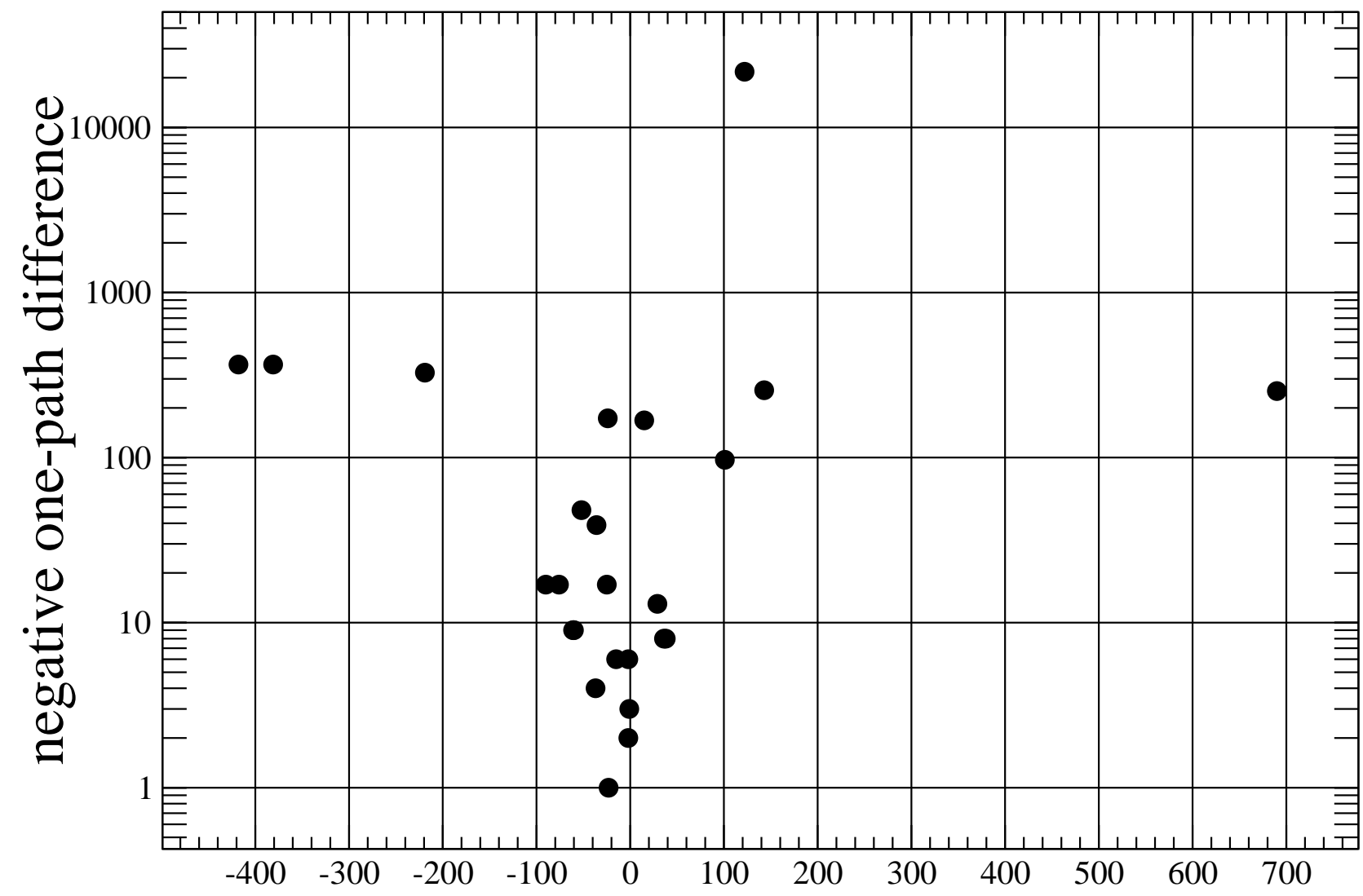

node difference

Figure 6: Differences between EA and MS. Plot of negative $P_{1}$ difference versus node difference.

\section{Acknowledgements}

We like to thank Görschwin Fey for providing the benchmark data of the MS algorithm. We also thank the referees for their helpful comments.

\section{Bibliography}

[1] ISCAS' 89 benchmark information. www.cbl.ncsu.edu/ CBL_Docs/ iscas89.html, 1997.

[2] F. Brglez, D. Bryan, and K. Kozminski. Combinational profiles of sequential benchmark circuits. In Int'l Symp. Circ. and Systems, pages 1929-1934, 1989.

[3] R. Bryant. Graph-based algorithms for Boolean function manipulation. IEEE Trans. on Comp., 35(8):677-691, 1986.

[4] R. Bryant. Binary decision diagrams and beyond: Enabling techniques for formal verification. In Int'l Conf. on CAD, pages 236-243, 1995.

[5] R. Drechsler. Evolutionary Algorithms for VLSI CAD. Kluwer Academic Publisher, 1998.

[6] R. Drechsler and N. Drechsler. Evolutionary Algorithms for Embedded System Design. Kluwer Academic Publisher, 2002.

[7] E. Dubrova and D. Miller. On disjoint covers and ROBDD size. In Pacific Rim Conference on Communications, Computers and Signal Processing, pages 162-164, 1999.

[8] G. Fey and R. Drechsler. Minimizing the number of paths in BDDs. In Symposium on Integrated Circuits and System Design, pages 359-364, 2002.
[9] G. Fey and R. Drechsler. A hybrid approach combining symbolic and structured techniques for disjoint sop minimization. In Workshop on Synthesis And System Integration of Mixed Information technologies (SASIMI), pages 54-60, 2003.

[10] N. Göckel, R. Drechsler, and B. Becker. GAME: A software environment for using genetic algorithms in circuit design. In Applications of Computer Systems, pages 240-247, 1997.

[11] D. Goldberg. Genetic Algorithms in Search, Optimization \& Machine Learning. Addision-Wesley Publisher Company, Inc., 1989.

[12] A. Mishchenko and M. Perkowski. Fast heuristic minimization of exclusive-sums-of-products. In Int'l Workshop on Applications of the Reed-Muller Expansion in Circuit Design, pages 242-250, 2001.

[13] I. Oliver, D. Smith, and J. Holland. A study of permutation crossover operators on the traveling salesman problem. In Int'l Conference on Genetic Algorithms, pages 224-230, 1987.

[14] S. Reda, R. Drechsler, and A. Orailoglu. On the relation between SAT and BDDs for equivalence checking. In Int'l Symp. on Quality Electronic Design, pages 394-399, 2002.

[15] R. Rudell. Dynamic variable ordering for ordered binary decision diagrams. In Int'l Conf. on CAD, pages 42-47, 1993.

[16] F. Somenzi. CUDD: CU Decision Diagram Package Release 2.3.1. University of Colorado at Boulder, 2001.

[17] Y. Ye and K. Roy. Efficient synthesis of AND/EXOR networks. In ASP Design Automation Conf., pages 539-544, 1997. 
Table 3: Best results of EA compared to MS

\begin{tabular}{|c|c|c|c|c|c|c|c|c|c|c|}
\hline \multirow[b]{2}{*}{ function } & \multirow[b]{2}{*}{ \#in } & \multirow[b]{2}{*}{ \#out } & \multicolumn{3}{|c|}{ MS } & \multicolumn{4}{|c|}{ EA } & \multirow{2}{*}{$\begin{array}{l}\Delta \\
\% \\
\end{array}$} \\
\hline & & & nodes & $P_{1}$ & $\mathrm{sec}$ & nodes & $P_{1}$ & $\mathrm{sec}$ & genr. & \\
\hline s1196 & 32 & 31 & 1523 & 2874 & 0.30 & 1142 & 2508 & 104.14 & 555 & 12.73 \\
\hline s1238 & 32 & 31 & 1523 & 2874 & 0.28 & 1105 & 2508 & 129.56 & 896 & 12.73 \\
\hline s1488 & 14 & 25 & 500 & 369 & 0.04 & 410 & 352 & 39.80 & 254 & 4.61 \\
\hline s1494 & 14 & 25 & 500 & 369 & 0.04 & 424 & 352 & 19.89 & 175 & 4.61 \\
\hline s208 & 18 & 9 & 62 & 53 & 0.02 & 62 & 53 & 25.02 & 235 & 0.00 \\
\hline s27 & 7 & 4 & 13 & 16 & $<0.005$ & 11 & 16 & 6.37 & 61 & 0.00 \\
\hline s298 & 17 & 20 & 91 & 70 & $<0.005$ & 88 & 70 & 17.54 & 165 & 0.00 \\
\hline s344 & 24 & 26 & 104 & 330 & 0.01 & 104 & 330 & 45.47 & 423 & 0.00 \\
\hline s349 & 24 & 26 & 104 & 330 & 0.01 & 104 & 330 & 37.10 & 345 & 0.00 \\
\hline s382 & 24 & 27 & 152 & 238 & $<0.005$ & 188 & 230 & 40.98 & 378 & 3.36 \\
\hline s386 & 13 & 13 & 158 & 61 & 0.01 & 121 & 57 & 18.08 & 171 & 6.56 \\
\hline s400 & 24 & 27 & 152 & 238 & 0.01 & 190 & 230 & 29.80 & 276 & 3.36 \\
\hline s444 & 24 & 27 & 154 & 243 & 0.01 & 183 & 230 & 34.17 & 313 & 5.35 \\
\hline s510 & 25 & 13 & 184 & 170 & 29.18 & 159 & 153 & 26.93 & 244 & 10.00 \\
\hline s526 & 24 & 27 & 153 & 162 & 0.01 & 138 & 156 & 40.59 & 375 & 3.70 \\
\hline s526n & 24 & 27 & 153 & 162 & 0.01 & 151 & 156 & 28.20 & 260 & 3.70 \\
\hline s641 & 54 & 42 & 768 & 1700 & 0.11 & 911 & 1444 & 216.99 & 1707 & 15.06 \\
\hline s713 & 54 & 42 & 768 & 1700 & 0.11 & 1458 & 1447 & 189.99 & 1498 & 14.88 \\
\hline s820 & 23 & 24 & 310 & 155 & 0.07 & 250 & 146 & 22.06 & 195 & 5.81 \\
\hline s832 & 23 & 24 & 310 & 155 & 0.06 & 249 & 146 & 30.21 & 269 & 5.81 \\
\hline alu4 & 14 & 8 & 621 & 1545 & 0.17 & 597 & 1372 & 40.02 & 322 & 11.20 \\
\hline b12 & 15 & 9 & 67 & 60 & $<0.005$ & 73 & 60 & 17.66 & 167 & 0.00 \\
\hline clip & 9 & 5 & 127 & 262 & 0.01 & 75 & 214 & 19.80 & 188 & 18.32 \\
\hline inc & 7 & 9 & 79 & 66 & 0.01 & 43 & 27 & 8.86 & 86 & 59.09 \\
\hline majority & 5 & 1 & 8 & 5 & $<0.005$ & 8 & 5 & 3.57 & 35 & 0.00 \\
\hline $\operatorname{misex} 1$ & 8 & 7 & 42 & 34 & $<0.005$ & 37 & 34 & 8.32 & 80 & 0.00 \\
\hline $\operatorname{misex} 2$ & 25 & 18 & 147 & 30 & 0.01 & 124 & 29 & 12.21 & 112 & 3.33 \\
\hline rd53 & 5 & 3 & 17 & 35 & $<0.005$ & 17 & 35 & 7.94 & 78 & 0.00 \\
\hline rd73 & 7 & 3 & 31 & 147 & $<0.005$ & 31 & 147 & 11.31 & 109 & 0.00 \\
\hline rd84 & 8 & 4 & 42 & 294 & $<0.005$ & 42 & 294 & 12.84 & 124 & 0.00 \\
\hline $\mathrm{sao} 2$ & 10 & 4 & 102 & 99 & 0.01 & 100 & 97 & 13.42 & 127 & 2.02 \\
\hline $\mathrm{t} 481$ & 16 & 1 & 35 & 1009 & $<0.005$ & 50 & 841 & 17.33 & 162 & 16.65 \\
\hline xor5 & 5 & 1 & 6 & 16 & $<0.005$ & 6 & 16 & 6.21 & 61 & 0.00 \\
\hline $5 x p 1$ & 7 & 10 & 45 & 82 & $<0.005$ & 44 & 79 & 10.62 & 102 & 3.66 \\
\hline 9sym & 9 & 1 & 25 & 148 & $<0.005$ & 25 & 148 & 11.28 & 109 & 0.00 \\
\hline $\begin{array}{l}\Sigma \\
\text { median } \\
\text { mean }\end{array}$ & & & 9076 & 16101 & 30.56 & 8738 & 14312 & 1304.28 & 10657 & $\begin{array}{l}3.66 \\
6.47 \\
\end{array}$ \\
\hline cordic & 23 & 2 & 49 & 30093 & 0.01 & 171 & 8332 & 84.60 & 747 & 72.31 \\
\hline $\operatorname{misex} 3$ & 14 & 14 & 946 & 2303 & 0.39 & 727 & 1976 & 43.42 & 325 & 14.20 \\
\hline seq & 41 & 35 & 1398 & 1841 & 4469.27 & 1499 & 1744 & 305.67 & 991 & 5.27 \\
\hline $\begin{array}{l}\Sigma \\
\text { mean }\end{array}$ & & & 2393 & 34237 & 4469.67 & 2379 & 12052 & 433.69 & 2063 & 30.59 \\
\hline
\end{tabular}

